УДК 519.86; 510; 514

ISSN 1995-5499

DOI: https://doi.org/10.17308/sait.2020.1/2575

Поступила в редакцию 20.10.2019

Подписана в печать 15.03.2020

\title{
О ГОМЕОМОРФНОСТИ ПРОСТРАНСТВ ДВУХ ФРАКТАЛЬНЫХ МОДЕЛЕЙ
}

\author{
(c) 2020 А. Г. Буховец ${ }^{\bowtie}$, М. В. Горелова, Е. А. Семин
}

\begin{abstract}
Воронежский государственный аграрный университет имени Императора Петра I ул. Мичурина, 1, 394087 Воронеж, Российская Федерация
\end{abstract}

\begin{abstract}
Аннотация. В статье приводятся итеративные алгоритмы, реализация которых позволяет получать фрактальные множества. Рассматриваются и последовательно сравниваются два алгоритма, которые реализуют классический подход, известный как «игра хаоса» с постоянными параметрами и подход, основанный на построении случайных разбиений на множестве элементов сходящегося ряда. Полученные результаты позволяют сделать вывод, что множество, получаемое в результате реализации второго подхода, является некоторым инвариантом для построенного предфрактального множества. Показано, что второй из рассмотренных подходов к построению фрактальных множеств, позволяет легко получать гомеоморфные предфрактальные множества. Анализируются топологические свойства аттракторов, полученных в ходе выполнения алгоритмов рандомизированных систем итерированных функций. Отмечается, что некоторые топологические свойства проще устанавливать в пространствах, полученных в ходе реализации второго подхода, и затем транслировать их на другие пространства. В работе представлены две модели биологических сообществ, имеющих стайный характер поведения животных. В качестве примеров, соответствующих интерпретациям двух приведенных в статье различных алгоритмов построения фрактальных множеств, рассматривается поведение медоносных пчел и пелагических рыб в период размножения и формирования стайных совокупностей. Показано, что сам характер размножения (генезис) и поведение особей этих биологических сообществ соответствуют различным алгоритмам процедур рандомизированных систем итерированных функций, которые были ранее рассмотрены. Наиболее заметны существенные различия в поведении этих стайных сообществ будут проявляться в поведении изолированных отдельных частей этих сообществ.
\end{abstract}

Ключевые слова: рандомизированные системы итерированных функций, фрактальные множества, аттрактор динамической системы, гомеоморфные множества, модели биологических сообществ.

\section{ВВЕДЕНИЕ}

В последнее время фрактальные модели получили широкое распространение в самых различных сферах деятельности: в моделировании рынков финансовых инструментов [1], в информационных технологиях [2], в задачах прогнозирования [9], в биоэкологии [21], в исследовании перколяционных процессов [23], в исследовании социально-экономиче-

Буховец Алексей Георгиевич e-mail: abuhovets@main.ru ских структур [25], в решении ряда физических задач и пр.

Использование фрактальных, а по сути дискретных моделей можно рассматривать как некоторое противопоставление континуальным (непрерывным) моделям, которое позволяет учитывать ряд особенностей динамических процессов при моделировании системных объектов. Наибольший интерес вызывают модели, представленные фрактальными множествами. Свойства фрактальных множеств, которые следует учитывать при решении практических задач, такие как са-

(i) Контент доступен под лицензией Creative Commons Attribution 4.0 License.

The content is available under Creative Commons Attribution 4.0 License. 
моподобие, несвязность, компактность и пр., оказались востребованными и находят в последнее время широкое применение в моделировании системных объектов. Указанные выше особенности этих множеств делают их полезными при описании динамических процессов, структурных характеристик классификационных задач, прогнозировании на основе временных рядов.

Отметим, что фрактальные модели хорошо воспроизводят такие свойства моделируемых объектов как случайный характер процессов и самоподобие траекторий. Однако некоторые свойства фрактальных моделей оказались на первый взгляд странными и неожиданными. Так, к примеру, некоторые фрактальные множества, генерируемые РСИФ, имеют, как было установлено, сингулярную функцию распределения. Этот факт ставит под сомнение использование вероятностных методов, базирующихся на оценках плотности распределения. Или, наличие гиперболических распределений, не обладающих конечными моментами, ставит под сомнение корректность использования статистических оценок этих моментов. И, наоборот, установление гомеоморфности моделируемых фрактальных множеств, позволяет использовать в дальнейшем более адекватный для конкретной задачи алгоритм.

Все это делает актуальным методы численного построения фрактальных множеств, исследование их свойств и разработку алгоритмов генерирования таких множеств, оценку их параметров и проведение сравнительных исследований с целью выбора адекватной модели.

\section{1. ОБЗОР МЕТОДОВ ПОСТРОЕНИЯ ФРАКТАЛЬНЫХ МНОЖЕСТВ ПОСРЕДСТВОМ \\ РАНДОМИЗИРОВАННЫХ СИСТЕМ ЛИНЕЙНЫХ ИТЕРИРОВАННЫХ ФУНКЦИЙ}

Системы итерированных функций (СИФ) представляют собой в самом общем виде определенный набор функций $\left\{f_{i}\right\}_{i=1}^{K}$, выполняемых в заданной последовательности.
В дальнейшем внимание в нашей статье будем уделено исключительно линейным функциям как наиболее простым и удобным в практических приложениях. В принципе функции $\left\{f_{i}\right\}_{i=1}^{K}$ могут быть самыми различными. Никаких ограничений на вид функций не накладывается. Но если функции дифференцируемые, то их можно линеаризовать, т. е. перейти к линейным приближениям. В этом случае будет получена система линейных рандомизированных функций.

По способу выполнения принято различать детерминированные (ДСИФ) и рандомизированные (РСИФ) алгоритмы реализаций. Эти термины, как свидетельствует Б. Мандельброт [1], были введены М. Барнсли (см., также [3]). Основное различие заключается в том, что в первом случае (ДСИФ) на каждом последующем шаге итерации выполняется весь набор функций, т. е. формируется множество

$$
X_{n+1}=f_{1}\left(x_{n}\right) \cup f_{2}\left(x_{n}\right) \cup \ldots \cup f_{K}\left(x_{n}\right) .
$$

Обозначается ДСИФ как $\mathrm{F} 1=\left(\mathbf{X} ; f_{i}, i=1, \ldots, K\right)$.

Во втором случае на множестве набора функций задается вероятностное распределение $\left\{f_{i} / p_{i}\right\}_{i=1}^{K}$ и за один шаг итерации выполняется только одна функция, выбранная в соответствии с заданным распределением $x_{n+1}=f_{i}\left(x_{n}\right)(i \in\{1,2, \ldots K\})[6,7]$. При использовании линейных СИФ генерируемое множество будет состоять всего из одной точки, координаты которой вычисляются по схеме [11],

$$
f_{j}\left(x_{n+1}\right)=\xi \cdot x_{n}+(1-\xi) \cdot z_{j}^{(n)} .
$$

В рассматриваемом нами случае набор представляют линейные функции, зависящие от параметров $\xi$ и $Z=\left\{z_{i}: i=1, \ldots, K\right\}$, а выбор функции определяется заданием вероятностей $p_{i}=p\left\{Z=z_{i}\right\}$. Очевидно, что $p_{i}>0, \quad \sum_{i=1}^{K} p_{i}=1$. Обозначение для РСИФ принято $\mathrm{F} 2=\left(\mathbf{X} ; f_{i} / p_{i}, i=1, \ldots, K\right)$.

Результатом выполнения СИФ в таком варианте является некоторое компактное множество, как правило, обладающее фрактальными свойствами [11]. Такой способ построения множества $\left\{X_{n}\right\} \in \mathbf{X}$ будем в дальнейшем обозначать F1 и называть X-пополнением.

В случае, если все функции итерируемой системы $\left\{f_{i}\right\}_{i=1}^{K}$ суть линейные функции, а $\xi-$ 
фиксированное значение параметра, которое одинаково для всех $\left\{f_{j}: j=1,2, \ldots, K\right\}$, т. е. выполнение РСИФ определяется формулой (1), построение аттрактора системы может быть произведено другим способом, который представляет собой некоторый аналог урновой схемы $[8,11]$. Для этой цели используется ряд

$$
\mu \sum_{i=1}^{\infty} \xi^{i}=1,
$$

где $\mu-$ нормировочная константа, связанная со значением параметра $\xi$ следующим образом: $\mu=\xi^{-1}(1-\xi)$. Элементы ряда (2) разносятся по $K$ классам, которые в классической литературе обычно принято называть урнами [13]. Это размещение осуществляется в соответствие с тем же распределением вероятностей $\left\{f_{i} / p_{i}\right\}_{i=1}^{K} \quad p_{i}>0, \sum_{i=1}^{K} p_{i}=1$. Получаемые в этом случае на каждом шаге суммы подмножеств ряда (2) записываются в виде кортежа (строки) $A_{l}=\left\{a_{l 1}, a_{l 2}, \ldots, a_{l K}\right\}$, причем каждый элемент $a_{l j}$ является суммой отобранных из указанного ряда (2) членов в соответствии с заданным вероятностным распределением. Очевидно, что получаемые в ходе выполнения процедуры значения удовлетворяют условиям: $a_{l j} \geq 0 ; \sum_{j=s}^{K} a_{l j}=1$. Полученные в результате такого выполнения совокупность строк $\mathbf{A}=\left\{A_{i}: i=1,2, \ldots N\right\}$ можно представить и записать в виде матрицы размером $N \times K$, где строки матрицы суть образованные ранее кортежи. При таком подходе окончательный результат выполнения процедуры будет получен в виде матричного произведения

$$
X=A Z,
$$

где $X-$ множество координат точек, называемых в дальнейшем предфракталом, $Z-$ множество точек (протофрактал), представленных матрицей размера $K \times P$; $A$ - матрица (кортежей) размера $N \times P$. Такой способ построения множества $\mathbf{X} \in\left\{X_{n}\right\}$ будем обозначать F2. На рис. 2 представлена коммутационная диаграмма построения протофрактального множества $\mathbf{X}$, подмножеством которого и является совокупность точек $X$. При этом правый верхний путь соответствует процедурам типа F1, а нижний левый процедурам типа F2.

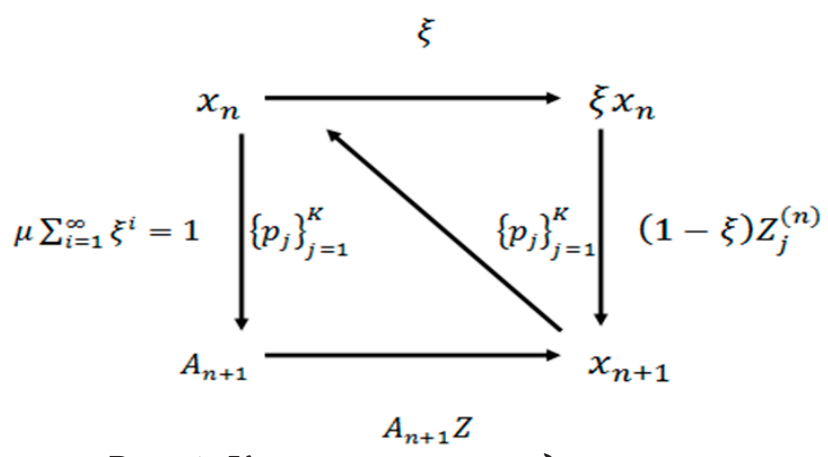

Рис. 1. Коммутативная диаграмма выполнения РСИФ

[Fig. 1. Commutative diagram of RSIF execution]

Будем в дальнейшем называть множество, получаемое в ходе выполнения процедуры $\mathrm{F} 1$, $\mathrm{X}$-пополнением (множества $Z$ ), а процедуры F2 - A-пополнением.

Отметим, что термин «пополнение» в этом контексте близок по смыслу к операции пополнения метрического пространства [10], которая в некотором смысле схожа с операциями генерирования новых объектов (точек) фрактальной структуры и сводится к включению предельных точек некоторой фундаментальной последовательности, в то время как точки, генерируемые фрактальными процедурами, не образуют фундаментальных последовательностей.

\section{2. ПРЕДСТАВЛЕНИЕ ОСНОВНЫХ РЕЗУЛЬТАТОВ ВЫПОЛНЕНИЯ РСИФ И МЕТОДОВ ИХ ИССЛЕДОВАНИЯ}

Для описания результатов выполнения РСИФ можно использовать любое из представленных ранее множеств, которые после введения соответствующей метрики получают статус метрического пространства. Во-первых, это пространство $\mathbf{X}$, которое образуют значения непосредственно измеряемых признаков объектов. Такое пространство в классификационных задачах принято называть признаковым [5]. Метрику в этом пространстве будем обозначать буквой $d$, формулы для её вычисления определяются уровнем измерения признаков. В частности, если все признаки объектов измерены в интервальной шкале, то стандартной функцией расстояния будет евклидова метрика, возможно взвешен- 
ная. Это пространство будем обозначать как $(\mathbf{X}, d)$. Элементы этого пространства $\mathbf{X}=\left\{X_{i}\right.$ : $i=1,2, \ldots\}$ непосредственно получаются при выполнении РСИФ в режиме F1. В случае простого евклидова расстояния $\mathbf{X}$ пространство является обычным $R^{K}-$ арифметическим $K$-мерным пространством.

Выполнение процедуры РСИФ в режиме F2 приводит к построению кольца $A$, которое после введения нормы

$$
\left\|A_{i}\right\|_{\xi}=\max _{j}\left|a_{i j}\right|_{\xi},
$$

где $\left|a_{i, j}\right|_{\xi}=\left|\mu\left(\xi^{s}+\xi^{s^{\prime}}+\xi^{s^{\prime \prime}}+\ldots+\xi^{s^{\prime \prime \prime}}+\ldots\right)\right|_{\xi}=\xi^{s}$, и соответствующей метрики $d_{\xi}\left(A_{s}, A_{t}\right)=$ $=\left\|A_{s}-A_{t}\right\|_{\xi}, \quad \forall s, t \in N$, становится метрическим, а точнее - ультраметрическим, пространством $\left(\mathbf{A}, d_{\xi}\right)($ см. $[11,15,30)$. Это пространство будем называть классификационным, поскольку в нем удобно представлять результаты классификации в виде сюръективного отображения множества А в фактор-множество $\mathbf{A} / \mathbf{S}$, где $\mathbf{S}=\left\{S_{j}: j=1,2, \ldots, K\right\}-$ совокупность полученных классов (кластеров). Отметим, что в классификационном пространстве многие алгоритмы кластерного анализа становятся тривиальными. Так, в силу иерархичности пространства $\left(\mathbf{A}, d_{\xi}\right)$ построение дендрограмм является тривиальной задачей, поскольку само ультраметрическое пространство иерархично. Проблема изолированности отдельных классов, получаемых с помощью иерархических алгоритмов, (т. н. «цепочный эффект»), нивелируется таким свойством классификационного пространства как несвязность. Итерационные алгоритмы, использующие в ходе реализации свойства сферических конструкций, становятся тривиальными поскольку любая точка внутри сферы может считаться ее центром. Таким образом, свойства пространства $\left(\mathbf{A}, d_{\xi}\right)$ позволяют получать некоторые классификационные разбиения не прибегая к использованию алгоритмов кластерного анализа. Однако при этом классификационные построения будут существенно зависеть от значения параметра $\xi$.

Для того, чтобы установить топологическую эквивалентность пространств $\left\{X_{n}\right\} \in \mathbf{X}$ и $\left\{A_{n}\right\} \in \mathbf{A}$ покажем, что существует непрерывное преобразование $F: \mathbf{A} \rightarrow \mathbf{X}$, а также и непрерывное обратное $F^{-1}: \mathbf{X} \rightarrow \mathbf{A} \quad$ [16]. В этом случае все топологические свойства одного пространства, такие как вполне разрывность, несвязность, компактность и др., будут передаваться от одного множества к другому.

Отображение $F: \mathbf{A} \rightarrow \mathbf{X}$ классификационного пространства в признаковое пространство является линейным (см. (3)), и, следовательно, непрерывным. Также непрерывным будет и обратное $F^{-1}: \mathbf{X} \rightarrow \mathbf{A}$ поскольку оно может быть представлено в виде преобразования Мура - Пенроуза (МП) [14]. В случае, когда ранг матрицы $Z$ определяется $\operatorname{rank}(Z)=\min \{K, P\}$, т. е. матрица полного ранга, имеем

$$
\tilde{A}=X\left(\left(Z^{T} Z\right)^{-1} Z^{T}\right) .
$$

В этом случае непосредственной подстановкой можно убедиться, что $\tilde{A}=A$, т. е.

$$
F^{-1}(\tilde{A})=F^{-1}(F(A))=A .
$$

При этом отметим, что преобразования $F$ и $F^{-1}$ будут биективными, и, очевидно, непрерывными.

В случае, когда матрица $Z$ не является матрицей полного ранга, обратное преобразование будет представлено в виде [14]

$$
\tilde{A}=X\left(\left(Z^{T} Z\right)^{-1} Z^{T}\right)+\left(I-\left(\left(Z^{T} Z\right)^{-1} Z^{T}\right) Z\right) Q,
$$

где $I-$ единичная матрица, а $Q-$ произвольная матрица соответствующего размера.

Первое слагаемое является ортогональной проекцией на ядро оператора $A$, а второе произвольным многообразием образа этого же оператора. За счет выбора $Q$ всегда можно добиться того, что вторе слагаемое будет равно нулю. Для этого достаточно положить $Q=A-\tilde{A}, \quad$ и тогда соотношение (4) $F^{-1}(F(A))=A$ будет выполнено. Таким образом преобразование $F$ является гомеоморфизмом для пространств $\mathbf{X}$ и $\mathbf{A}$ с естественной топологией [16]. 


\section{3. ОБСУЖДЕНИЕ ПОЛУЧЕННЫХ РЕЗУЛЬТАТОВ \\ 3.1. Теоретические аспекты}

Пусть $X=A Z$, и протофрактал $Z$ гомеоморфно преобразован в множество $Z^{*}$. Тогда, посредством той же матрицы можно построить предфрактал $X^{*}=A Z^{*}$. При этом, как указано выше, $X^{*}$ будет гомеоморфен $A$. В свою очередь $X$ также гомеоморфен $A$. Учитывая свойство транзитивности гомеоморфизма как преобразования [10], будет справедливо утверждение о гомеоморфности множеств $X \cong X^{*}$.

Полученные результаты позволяют сделать вывод, что А-пополнение является некоторым инвариантом построения фрактала посредством РСИФ классификационной задачи. В рамках A-пополнения удобно строить классификационные схемы, анализировать результаты классификации, исследовать такие топологические свойства как компактность, связность, совершенность. В то же время, изменяя конфигурацию точек протофрактала $Z$ с помощью топологического преобразования можно получать в признаковом пространстве различные структуры многомерных данных, являющихся топологически эквивалентными. Так на рис. 2 представлены две различные геометрические фигуры, соответствующие одному и тому же массиву $A$, но имеющие различные конфигурации протофрактала.

Анализируя результаты выполнения процедур РСИФ, можно отметить следующие характерные для процедур F2 особенности:

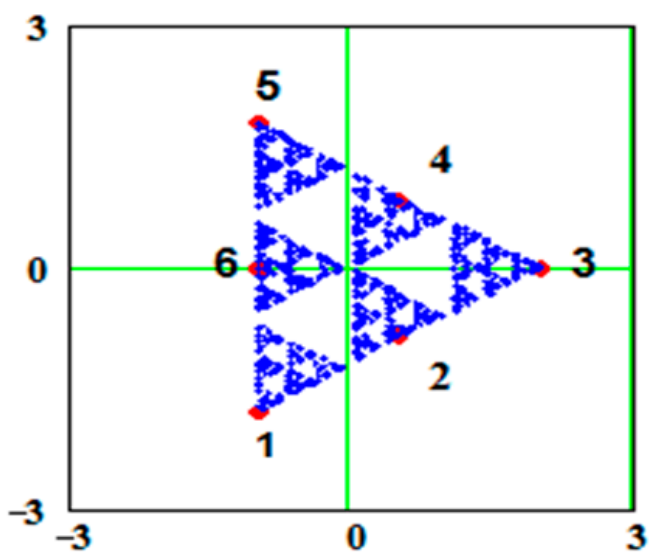

Рис. 2. Гомеоморфные структуры аттракторов РСИФ

[Fig. 2. However, the structure of the attractors of RSIF]
- Изменение расположения точек протофрактала $(1,2,3,4,5,6)$, выполненное с помощью топологического преобразования, при сохранении значений массива $A$ позволяет получать различные конфигурации;

- Структура массива $A$ остается неизменной (инвариантной), т. е. содержит шесть различных классов, полученных при одном и том же значении параметра $\xi=0,3(\mu=2,3)$.

В классификационном пространстве эти массивы, представленные матрицей $A$, будут одними и теми же, а в признаковом пространстве $X$, как нетрудно видеть, будут иметь различную структуру, но при этом будут сохранять число классов, равное шести. Массивы, полученные с помощью одной и той же матрицы $A$, формируют различные, гомеоморфные структуры (см. рис. 2) При этом хорошо заметно, что в признаковом пространстве такие топологические свойства, как связность множеств, компактность, подобие кластеров, равномощность дискретных множеств сохраняются.

Проблемы гомеоморфизма рассматривались в $[17,18]$ где использовалось, т. н. кодовое пространство $\Omega_{\{0,1, \ldots, K-1\}}$ (code space). Это пространство получают за счет биективного отображения $\phi: Z \rightarrow \Omega_{\{0,1, \ldots, K-1\}}$. В теореме 4.14 .5 утверждается, что множества аттракторов являются гомеоморфными, если они обладают одинаковой структурой кодового пространства.

Представленные результаты согласуются с теоремой Александрова [12], которая утверждает, что два вполне несвязных ком-

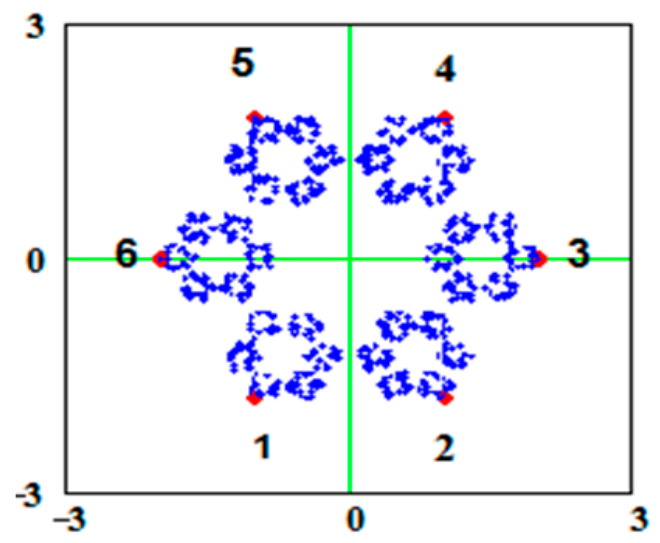



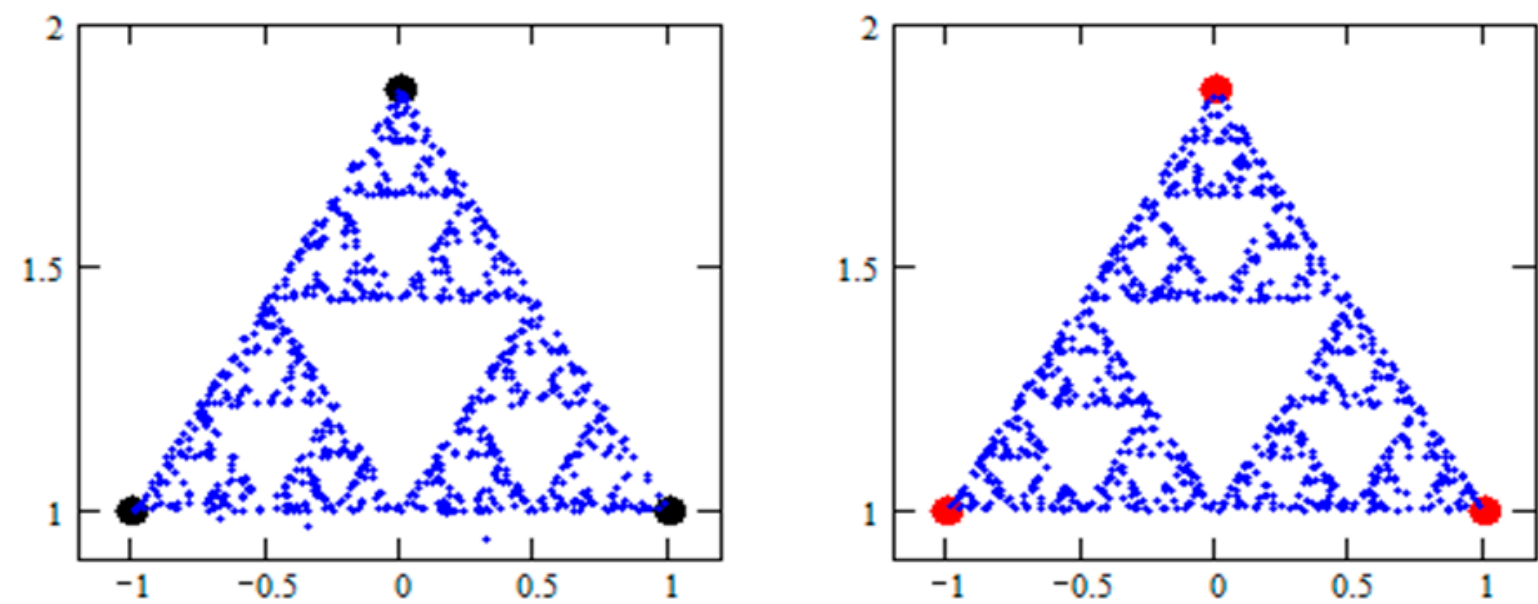

Рис. 3. Попробуйте определить какой рисунок каким алгоритмом получен.

(Ответ можно найти в Заключении)

[Fig. 3. Try to determine which drawing was obtained by which algorithm.

(The answer can be found in the Conclusion)]

пакта (одинаковой мощности) гомеоморфны. Но для нас был важен не только сам факт существования гомеоморфизма двух множеств, как это было установлено ранее, но и возможность в явной форме получить вид биективного преобразования. Этот результат в дальнейшем позволяет получить статистические оценки параметров РСИФ и аппроксимировать протофракталы [9].

Замечание. Визуально результаты процедур F1 и F2 практически не отличимы друг от друга, в чем легко убедиться, сравнивая соответствующие геометрические построения [24]. Однако, при этом имеются и некоторые различия, о которых следует упомянуть ввиду использования их в практической деятельности.

Так, на первых шагах результаты выполнения процедуры F1 зависят от начального значения $X_{0}$. В дальнейшем, как легко можно показать [4], эта зависимость в значительной степени уменьшается, что позволяет пренебречь ею. В случае выполнения процедуры F2 такая зависимость полностью отсутствует. Если анализировать получаемое множество данных $\left\{X_{n}\right\} \in \mathbf{X}$ с точки зрения последовательного появления значений $X_{n}$, т. е. рассматривать их как некоторый временной ряд, различие будет проявляться в том, что данные, полученные посредством процедуры F1 имеют значение коэффициента автокорреляция равное, с учетом стохастического харак- тера, значению параметра $\xi$. В случае реализации процедуры F2 оценка коэффициента автокорреляции будет статистически незначимой, т.е. коэффициент автокорреляции будет равным нулю. Это легко объясняется независимым характером построения кортежа $A_{l}=\left\{a_{l 1}, a_{l 2}, \ldots, a_{l K}\right\}$. Эти свойства следует учитывать при решении практических задач [9].

В заключение отметим, что топологические отображения, т. е. гомеоморфизмы не сохраняют метрические свойства множеств, такие, например, как фрактальную размерность [2].

\section{2. Примеры биологических систем, имеющих сходные феноменологические признаки, но разные механизмы генезиса сообществ}

Завершая рассмотрение алгоритмов выполнения РСИФ отметим, что можно указать образцы природных систем биологического происхождения, в которых принципы генерирования как самих систем, так и их функционирования соответствуют различным процедурам выполнения алгоритмов РСИФ. В качестве примеров обратимся к моделированию стайного поведения животных. По определению стаей является «любая подвижная, обычно временная группировка насекомых, рыб и птиц, изредка млекопитающих» [26]. В дальнейшем мы будем использовать 
именно этот термин, хотя в специализированной литературе предпочтение часто отдается синонимам, традиционно закрепленным за некоторыми видами животных, например: стадо (буйволов, оленей), косяк (рыбы), рой (пчел, комаров), стая (птиц) и т. п. Поскольку нас будет интересовать исключительно аспект математического моделирования группирования и поведения отдельных индивидов, входящих в состав сообществ, то мы в дальнейшем будем использовать термин «стая» для всех этих проявлений характерного поведения.

Стайный образ жизни, является одной из форм социального поведения животных, который позволяет им успешно конкурировать с другими видами. Стайные формы существования способствуют не только снижению уязвимости и увеличению степени защиты отдельных индивидов от хищников, но и приобретению входящих в состав стаи многих жизненно важных преимуществ при питании, миграциях, размножении [26].

Стая может быть представлена как системный объект [20]. Укажем наиболее важные с точки зрения системного анализа свойства стайных объединений, которые подтверждают этот тезис:

- Взаимодействие особей, входящих в состав стаи; (взаимодействие особей внутри стаи более сильное, чем с особями, находящимися вне стаи);

- Устойчивость как способность противостоять внешним воздействиям с целью самосохранения. Отметим особый тип устойчивости - структурную устойчивость, которая сохраняется за счет замены элементов системы;

- Эмерджентность - т. е. несводимость свойств системы к свойствам, присущим образующим систему элементов;

- Целостность - взаимодействие с внешней средой как некоторое целое.

Таким образом стая может рассматриваться как системный объект, обладающий всеми указанными выше свойствами.

В качестве одного из примеров рассмотрим формирование и дальнейшее поведение семей медоносных пчел $[27,28]$, и сравним с поведением рыб [22]. Этим биологическим объектам, как известно, свойственно соци- альное поведение, которое носит стайный характер на определенных этапах их жизненного цикла.

Так, хорошо известно, что все указанные выше биологические сообщества: пчелы, муравьи, комары, бабочки, рыбы и птицы в ходе своего существования на определенных стадиях жизненного цикла проявляют «стайный» характер (тип) поведения, выражающийся в некотором «хаотическом» перемещении особей в пространстве при движении всей совокупностей как единого целого. Другими словами, эти сообщества можно наблюдать в виде стай или роев. И в этом плане характер их поведение (еще раз почеркнем на определенной стадии развития) совпадают: они образуют биологическую систему [19], отвечающую перечисленным ранее основным признакам, определяющим систему. В качестве модели такого поведения мы предлагаем использовать математические модели данных, полученных с помощью РСИФ. Такая модель будет отражать стайный характер поведения при условии сохранения общей численности сообщества, который можно представить в виде предфрактала, т. е. совокупности сгенерированных точек, а также позволит смоделировать способ генерирования популяции.

Семьи медоносных пчел представляют ярко выраженные общественные колонии (семьи), в каждой из которых имеется одна пчелиная матка, тысяч рабочих пчёл и на определенных стадиях развития - трутни [27]. Матка - это единственная особь пчелиной семьи, которая откладывает потомство и таким образом отвечает за воспроизводство семьи. Для того, чтобы самка стала плодной, т.е. способной приносить потомство, ее осеменяют 7-10 трутней (полиандрия). Отметим, что количество трутней может быть различным и доходить по данным различных авторов до 23, но с точки зрения нашей модели важным является тот факт, что их количество конечно, как и число точек протофрактала $Z$.

Рабочие пчелы появляются из отложенных маткой яичек, которые в ходе своего развития становятся личинками. Таким образом, 
хромосомы пчелиной семьи передаются от трутней и матки каждой новой особи. Другими словами, этот процесс может быть представлен уравнением $x_{n+1}=\xi \cdot x_{n}+(1-\xi) \cdot z_{j}^{(n)}$, где $x_{n+1}$ характеризует новое поколение особей пчелиной семьи, $z_{j}^{(n)}-$ роль $j$-го трутня в формировании последующего поколения особей, отношение величин $\xi /(1-\xi)$, где параметр $0<\xi<1$ определяет вклад предшествующих поколений в новое, передаваемого через матку и трутней новым особям. Такой подход в некоторой смысле аналогичен методике генетических алгоритмов (на этапах отбора, скрещивания и мутации), где особи нового поколения имеют хромосомы, содержащие часть генов от каждого из родителей. Однако, в отличие от генетических алгоритмов, фитнесс-функция в случае РСИФ не рассматривается [31], а предпочтение отдается партнеру, например, имеющему наибольшее значение вероятности $P_{j}=p\left\{Z=z_{j}\right\}$.

По характеру поведения для такого сообщества (популяции) наиболее подходящей моделью будет процедура F1 рандомизированной системы, представленная уравнением (2). Возможно, что вместо одного значения $x_{n}$ будет иметь смысл рассматривать одновременно несколько значений $x_{n}$, или вообще перейти к механизму детерминированных систем итерированных функций (ДСИФ). Выбытие особей можно моделировать как исключение из рассмотрения ранее сгенерированных точек.

Кроме этого отметим также тот момент, что ни матка, ни рабочие пчёлы, ни трутни отдельно существовать не могут, и не могут самостоятельно образовывать новую семью, точно так же как нельзя реализовать процедуру F1, если не задан хотя бы один из параметров $\xi, Z=\left\{z_{n}\right\}, x_{n}$, являющихся необходимыми элементами РСИФ.

Таким образом, конфигурационное пространство стайного поведения медоносных пчел может быть представлено в рамках РСИФ процедурой F1.

В качестве примера другой модели рассмотрим поведение стайных рыб. Из животных с преобладанием однородных стай без лидеров наиболее характерными являются рыбы. Стайный образ жизни широко представлен среди пелагических рыб. Он присущ большому числу видов, различающихся по систематическому положению, биологическим характеристикам и образу жизни, населяющих морские и пресные воды, крупные и небольшие водоёмы в высоких и низких широтах, обитающих в условиях потока или в стоячих водах [22]. Стайные рыбы, как правило, являются раздельнополыми. Размножение стайных рыб происходит половым путем, как правило наружным способом: в период нереста самки выметывают икринки, а самцы - молоки, содержащие сперматозоиды. Оплодотворение икры происходит в воде.

Для такого поведения при размножении наиболее подходящей моделью будет реализация F2 процедуры РСИФ. Характерными признаками этой модели является независимое появление примерно одновременно большого числа отложенных яйцекладок, которые формируют в дальнейшем совокупность рыбной популяции. Другими словами, период нереста может быть представлен в модели как способ формирования матриц $X$ и $A$ в процедуре F2. Роль самок сводится к формированию матрицы $A$, а формирование матрицы $Z$ выполняют самцы. Взаимодействие икры и молок, приводящее к появлению молоди рыбной стаи, будет представлено в виде произведения $X=A Z$. Аналогичное поведение (в плане независимости и примерной одновременности) при размножении отмечается у лягушек.

При этом, как уже было отмечено ранее, визуальное проявление стайного характера поведения вновь появившихся особей, как пчелиного роя, так и рыбной молоди феноменологически очень похоже друг на друга. То же самое можно отметить и в геометрических результатах выполнения алгоритмов РСИФ для F1 и F2, внешне они на глаз трудно неразличимы.

Рассмотренные модели стайного поведения животных отражают и некоторые другие специфические особенности функционирования стай, различающихся моделями формирования. Но если рассмотреть процессы образования (генезиса) этих биологических сообществ (см. $[22,27])$, то можно найти за- 
метные отличия. Так, пчелы или муравьи [29] имеют единого родителя: «матка» у пчел, у муравьев за воспроизводство популяции отвечают одна или несколько яйцекладущих самок - цариц или господствующих муравьев. Однако при внешнем сходстве образа жизни существования этих систем по-разному проявляется во временном аспекте.

Кроме этого следует отметить также, что в биологических сообществах, характеризующихся выполнением F1 отмечаются элементы иерархии. Так, в зависимости от возраста особи сообществ, организованных определенным образом, выполняют различные по значимости функции. Бывают пчелы, которые строят соты, пчелы, которые выполняют функции сбора нектара, более старые пчелы выполняют сторожевую или охранную функцию. Аналогичным образом распределяются роли среди муравьев в муравейнике. Это свойство иерархичности хорошо заметно в процедурах СИФ, особенно в детерминистских вариантах. И наоборот, такая строгая иерархия отсутствует в рыбьих стаях, что характерно для реализации алгоритма F2.

Но основные различия проявляется в поведении изолированной части системы. Так, изолированная часть системы пчел, (муравьев, ос, шершней), лишенные матки, обречена на вымирание: время существования такого подмножества ограничено временем существования отдельных особей. Для обособленной части рыбной популяции (птиц, комаров) при наличии ресурсов система может восстановиться и продолжать существовать во времени, и даже воспроизводиться как система. Такие различия в поведении биологических, социально-экономических систем являются следствием различий в генезисе элементов, образующих эти системы.

\section{ЗАКЛЮЧЕНИЕ}

В представленной работе были рассмотрены способы численного моделирования фрактальных множеств с помощью линейных рандомизированных систем итерированных функций. Один из способов представляет собой классический подход, известный как «игра хаоса», другой связан с построениями случайных разбиений на множестве элементов сходящегося ряда и образованием некоторой матрицы, элементы которой представляют собой суммы разнесенных по различным классам построенного разбиения элементов ряда. Полученные при этом предфрактальные множества обладают сходными топологическими свойствами. Второй способ позволяет сравнительно легко получать гомеоморфные предфрактальные множества. Кроме этого, полученная матрица легко может быть использована в построении самоподобных реплик аттрактора РСИФ [5].

Наличие двух различных моделей построения аттракторов фрактальных множеств может служить интерпретацией генезиса биологических систем, представляющих стайное поведение животных. В качестве примеров в статье анализируются поведение медоносных пчел и пелагических рыб. Было показано, что характерные черты их поведения в период размножения и формирования стайных совокупностей соответствует различным алгоритмам генерирования фрактальных множеств. Эти результаты позволяют при наличии соответствующего эмпирического материала моделировать размножение и дальнейшее поведение биологических совокупностей особей, носящих стайный характер.

И последнее: на рис. 3 левое изображение получено посредством процедуры F1, а правое - F2.

\section{КОНФЛИКТ ИНТЕРЕСОВ}

Авторы декларируют отсутствие явных и потенциальных конфликтов интересов, связанных с публикацией настоящей статьи.

\section{СПИСОК ЛИТЕРАТУРЫ}

1. Мандельброт, Б. Б. Фракталы и хаос. Множество Мандельброта и другие чудеса / Б. Б. Мандельброт. - М.-Ижевск : НИЦ «Регулярная и хаотическая динамика», 2009. - 392 с.

2. Кроновер, Р. М. Фракталы и хаос в динамических системах / Р. М. Кроновер. - М. : ТЕХНОСФЕРА, 2006. - 488 c. 


\section{А. Г. Буховеи, М. В. Горелова, Е. А. Семин}

3. Barnsley, M. F. Fractals everywhere / M. F. Barnsley. - Boston : Academic Press, 1988. - 394 p.

4. Bukhovets, A. G. Modeling of fractal data structures / A. G. Bukhovets, E. A. Bukhovets // Automation and Remote Control. - 2012. - V. 73, No. 2. - P. 381-385.

5. Буховеи, А. Г. Структура аттрактора рандомизированных линейных систем итерированных функций / А. Г. Буховец, Т. Я. Бирючинская // Вестник Воронеж. гос. ун-та. Сер. Системный анализ и информационные технологии. - 2016. - № 2. - С. 5-10.

6. Shrivastava, S. C. Iterated Function System / S. C. Shrivastava, Priyanka, J. Chandra // 2nd International Seminar On "Utilization of Non-Conventional Energy Sources for Sustainable Development of Rural Areas ISNCESR'16 17th \& 18th March 2016, P. 456-458.

7. On the chaos game of iterated function system / Barrientos P.G. [et al.] // Topological Methods in Nonlinear Analysis. - 2017. - V. 49, No. 1. - P. 105-132.

8. Буховеи, А. Г. Моделирование фрактальных свойств системных объектов / А. Г. Буховец, Т. Я. Бирючинская // Вестник Воронеж. гос. ун-та. Сер. Системный анализ и информационные технологии. - 2011. - № 2. - С. 22 26.

9. Буховеи, А. Г. Современные подходы и методы в прогнозировании урожайности отдельных видов зерновых культур: монография / А. Г. Буховец, Е. А. Семин, Т. Я. Бирючинская. - Воронеж : ФГБОУ ВПО ВГАУ, 2016 - 226 c.

10. Колмогоров, A. Н. Элементы теории функций и функционального анализа / А. Н. Колмогоров, С. В. Фомин. - М. : Наука, 1976. - 544 c.

11. Bukhovets, A. G. Ultrametric properties of the attractor spaces for random iterated linear function systems / A. G. Bukhovets, P. V. Moskalev // Journal of Physics Conference Series 973(1):012028, DOPI 10.1088/17426596/973/1/012028.

12. Хаусдорф, Ф. Теория множеств / Ф. Хаусдорф. - М. : 1937. - 304 с.

13. Феллер, В. Ведение в теорию вероятностей и ее приложения. Т. 1 / В. Феллер. - М. : Мир, 1984. - 527 с.
14. Воеводин, В. В. Энциклопедия линейной алгебры / В. В. Воеводин, Вл. В Воеводин. - СПб. : БХВ-Петербург, 2006. - 544 с.

15. Хренников, А. Ю. Моделирование процессов мышления в р-адических системах координат / А. Ю. Хренников. - М. : ФИЗМАТЛИТ, 2004. - 296 с.

16. Engelking, R. General topology / R. Engelking. - Warsawa : PWN, 1977.

17. Barnsley, M. F. Superfractals / M. F. Barnsley. - Cambridge : Cambridge University Press, 2006. - 464 p.

18. Hutchinson, J. E. Fractals and self-similarity/ J. E. Hutchinson // Indiana Univ. Math. J. 1981. - V. 30. - P. 713-747.

19. Шрейдер, Ю. А. Системы и модели / Ю. А. Шрейдер, А. А Шаров. - М. : Радио и связь, 1982. - 152 с.

20. Флейшман, Б. С. Основы системологии / Б. С.Флейшман. - М. : Радио и связь, 1982. - 368 c.

21. Гелиашвили, Д. Б. Фракталы и мультифракталы в биоэкологии: монография / Гелиашвили [и др.]. - Н. Новгород : Изд-во Нижегородского госуниверситета, 2013. - 370 с.

22. Касумян, А. О. Эволюция стайного поведения рыб / А. О. Касумян, Д. С. Павлов // Вопросы ихтиологии. - 2018. - Т. 58, № 5. C. 534-543.

23. Москалев, П. В. Перколяционное моделирование пористых структур / П. В. Москалев. - Москва: URSS, 2018 - 240 с.

24. «RIFS» Рандомизированная система итерированных функций: свидетельство о регистрации программы №PR14002 ФАП СО РАН / П. В. Москалев, А. Г. Буховец, Т. Я. Бирючинская. Дата регистрации: 10.04.2014; URL: http://fap.sbras.ru/ node /4077.

25. Иванов, С. А. Основы теории случайных кластерных процессов и их практическое применение / С. А. Иванов. - М. : ЛЕНАНД, 2017. - 224 c.

26. Типы сообществ животных. - Режим доступа: http://biofile.ru/bio/1517.html (Дата обращения: 18.10.2019).

27. Организация пчелиного улья. Haceление и ресурсы. - Режим доступа: https:// pikabu.ru/story/organizatsiya_pchelinogo_ulya_ naselenie_i_resursyi_6267762 (Дата обращения 12.10.2019). 
28. Социальное поведение медоносных пчёл. - Режим доступа: http://apiary.su/ biologiya-pchelinoj-semi/sotsialnoe-povedeniemedonosnyh-pchyo/ (Дата обращения: 18.10.2019).

29. Муравьи и ихняя жизнь. - Режим доступа: https://dynozavri.ru/nasekomie/ muravi_i_ixnyaya_zhizn_chast_1/ (Дата обращения 12.10.2019).
30. Аветисов, B. А. Ультраметрическое случайное блуждание и динамика белковых молекул / В. А. Аветисов, А. Х. Бикулов, А. П. Зубарев. // Труды мат. ин-та им. В. А. Стеклова. - 2014. - Т. 285. - С. 4-24.

31. Goldberg, D. E. Genetic Algorithm in Search, Optimization and Machine Learning / D. E. Goldberg - N. York : Addison - Wesley, 1989. - $405 \mathrm{p}$.

Буховец Алексей Георгиевич - д-р техн. наук, профессор, профессор кафедры экономического анализа, статистики и прикладной математики Воронежского государственного аграрного университета имени императора Петра I».E-mail: abuhovets@main.ru

ORCID iD: https://orcid.org/0000-0002-6614-044X

Горелова Марина Владимировна - канд. экон. наук, доцент кафедры экономического анализа, статистики и прикладной математики Воронежского государственного аграрного университета имени императора Петра I. E-mail: gorelo.marina@yandex.ru

ORCID iD: https://orcid.org/0000-0003-0335-5934

Сёмин Евгений Александрович - канд. экон. наук, доцент кафедры экономического анализа, статистики и прикладной математики Воронежского государственного аграрного университета имени императора Петра I. E-mail: 113ghz@mail.ru

ORCID iD: https://orcid.org/0000-0002-4674-6532

DOI: https://doi.org/10.17308/sait.2020.1/2575

Received 20.10.2019

Accepted 15.03.2020

ISSN 1995-5499

\title{
HOMEOMORPHIC SPACES OF TWO FRACTAL MODELS
}

\author{
(C) 2020 A. G. Bukhovets ${ }^{\varpi}$, M. V. Gorelova, E. A. Semin \\ Voronezh State Agricultural University \\ 1, Mitchurina Str., 394087 Voronezh, Russian Federation
}

\begin{abstract}
The article describes the iterative algorithms used to obtain fractal sets. It analyses and compares two algorithms: one implementing a classical approach, known as the chaos game, with constant parameters, and the other implementing an approach based on the construction of random partitions based on a set of elements of a convergent series. The results of the analysis allowed us to conclude that the set obtained using the second approach is an invariant of the constructed prefractal set. It is shown that using the second approach to the construction of fractal sets we can easily obtain homeomorphic prefractal sets. The topological properties of attractors obtained by constructing iterated random function systems are analysed. The article demonstrates that it is easier to determine some topological properties in the spaces obtained during the implementation of the second approach, and then map them on to the other spaces. The paper presents two models of biological communities characterised by the gregarious behaviour of animals. The behaviour of honeybees and pelagic fish during breeding and the formation of gregarious communities is
\end{abstract}

Bukhovets Aleksey G.

e-mail: abuhovets@main.ru 


\section{А. Г. Буховеи, М. В. Горелова, Е. А. Семин}

considered as an example corresponding to the interpretations of the two different algorithms of fractal set construction considered in the article. It is demonstrated that the very nature of reproduction (genesis) and behaviour of individuals of these biological communities corresponds to different algorithms of the previously considered iterative random function systems. The differences in the behaviour of these gregarious communities are most noticeable in the behaviour of isolated individual parts of these communities.

Keywords: iterated random function system, fractal sets, attractor in a dynamical system, homeomorphic sets, models of biological communities.

\section{CONFLICT OF INTEREST}

The authors declare the absence of obvious and potential conflicts of interest related to the publication of this article.

\section{REFERENCES}

1. Mandelbrot B. B. Fractals and Chaos The Mandelbrot Set and Beyond. Moscow- Izhevsk, «Regulyarnaya i khaoticheskaya dinamika», 2009. 392 p. DOI: 10.1007/978-1-4757-4017-2.

2. Crownover R. M. Fractals and Chaos in dynamic systems. Fundamentals of the theory. Moscow, TEKHNOSFERA. 2006. 488 p.

3. Barnsley M. F. Fractals everywhere. Boston, Academic Press. 1988. 394 p.

4. Bukhovets A. G. and Bukhovets E. A. Modeling of fractal data structures. Automation and Remote Control, 2012. Vol. 73, No. 2. P. 381-385.

5. Bukhovets A. G. and Biryuchinskay T. Y. The attractor structure of randomized linear systems of iterated functions. Vestnik VSU, System Analysis and Information Technologies. 2016. No. 2. P. 5-10.

6. Shrivastava S. C., Priyanka, Chandra J. Iterated Function System. 2nd International Seminar On "Utilization of Non-Conventional Energy Sources for Sustainable Development of $\mathrm{Ru}$ ral Areas ISNCESR'16 17th \& 18th March 2016. P. 456-458.

7. Barrientos P. G. [et al.] On the chaos game of iterated function system. Topological Methods in Nonlinear Analysis. V. 49, No. 1. 2017. P. 105132.

8. Bukhovets A. G. and Biryuchinskay T. Y. Modeling fractal properties of system objects. Vestnik VSU, System analysis and information technologies. 2011. No. 2. P. 22-26.

9. Bukhovets A. G., Semin E. A. and Biryuchinskay T. Y. Modern approaches and methods in forecasting the yield of certain types of grain crops: monography. Voronezh, VSAU. 2016. $226 \mathrm{p}$.

10. Kolmogorov A. N. and Fomin S. V. Elements of the theory of functions and functional analysis. Moscow, Nauka. 1976. 544 p.

11. Bukhovets A. G. and Moskalev P. V. Ultrametric properties of the attractor spaces for random iterated linear function systems. Journal of Physics Conference Series 973(1):012028, DOPI 10.1088/1742-6596/973/1/012028.

12. Hausdorff F. Theory of sets. Moscow, 1937. $304 \mathrm{p}$.

13. Feller $V$. An Introduction to Probability Theory and its Applications. Vol. 1. Moscow, Mir. $1984.527 \mathrm{p}$.

14. Voevodin V. V. and Vojvodin Vl. V. Encyclopedia of Linear Algebra. Saint-Petersburg, BHV-Petersburg. 2006. 544 p.

15. Khrennikov A. Yu. Modeling thinking processes in p-adic coordinate systems. Moscow, FIZMATLIT. 2004. 296 p.

16. Engelking R. General topology. Warsawa, PWN, 1977.

17. Barnsley M. F. Superfractals. Cambridge, Cambridge University Press. 2006. 464 p.

18. Hutchinson J. E. Fractals and self-similarity. Indiana Univ. Math. J. 1981. V. 30. P. 713-747.

19. Schreider Yu. A. and Sharov A. A. Systems and models. Moscow, Radio and communications. $1982.152 \mathrm{p}$.

20. Fleishman B. S. Fundamentals of systemology. Moscow, Radio and communications. $1982.368 \mathrm{p}$.

21. Gelashvili D. B. et al. Fractals and multifractals in Bioecology: monography. Nizhny Novgorod, Nizhny Novgorod State University. 2013. 370 p.

22. Kasumyan A. O. and Pavlov D. S. The evolution of fish gregarious behavior. Questions of ichthyology. 2018. V. 58, No 5. P. 534-543. 
23. Moskalev P. V. Percolation modeling of porous structures. Moscow, URSS. 2018. 240 p.

24. Moskalev P. V., Bukhovets A. G. and Biryuchinskay T. Y. «RIFS» Randomized system of iterated functions: certificate of registration of the program No PR14002 FAP SB RAS. Registration date: 10.04.2014; URL: http://fap.sbras.ru/node /4077.

25. Ivanov S. A. Fundamentals of the theory of random cluster processes and their practical application. Moscow, LENAND. 2017. 224 p.

26. Types of animal communities. Available at: http://biofile.ru/bio/1517.html (accessed: 18.10.2019).

27. Organization of a bee hive. Population and resources. Available at: https://pikabu.ru/ story/organizatsiya_pchelinogo_ulya_naselenie_i_resursyi_6267762 (accessed: 12.10.2019).
28. Social behavior of honeybees. Available at: http://apiary.su/biologiya-pchelinoj-semi/ sotsialnoe-povedenie-medonosnyh-pchyo (accessed: 18.10.2019).

29. Ants and their life. Available at: https:// dynozavri.ru/nasekomie/muravi_i_ixnyaya_ zhizn_chast_1 (accessed: 12.10.2019).

30. Avetisov V. A., Bikulov A. H. and Zubarev A. P. Ultrametric random walk and dynamics of protein molecules. Works of Math. Institute named after V. A. Steklova. 2014. V. 285. P. 4-24.

31. Goldberg D. E. Genetic Algorithm in Search, Optimization and Machine Learning. New York, Addison - Wesley. 1989. 405 p.

Bukhovets Aleksey G. - DSc in Technical Sciences, Professor, Department of Economic Analysis, Statistics, and Applied Mathematics, Voronezh State Agricultural University named after Emperor Peter I. E-mail: abuhovets@mail.ru

ORCID iD: https://orcid.org/0000-0002-6614-044X

Gorelova Marina V. - PhD in Economics, Associate Professor, Department of Economic Analysis, Statistics, and Applied Mathematics, Voronezh State Agricultural University named after Emperor Peter I. E-mail: gorelo.marina@yandex.ru

ORCID iD: https://orcid.org/0000-0003-0335-5934

Semin Evgeniy A. - PhD in Economics, Associate Professor, Department of Economic Analysis, Statistics, and Applied Mathematics, Voronezh State Agricultural University named after Emperor Peter I. E-mail: 113ghz@mail.ru ORCID iD: https://orcid.org/0000-0002-4674-6532 